\title{
Increased mortality during early embryonic development after in-vitro fertilization of rat oocytes
}

\author{
B. C. Vanderhyden, A. Rouleau, E. A. Walton and D. T. Armstrong \\ M.R.C. Group in Reproductive Biology, and Departments of Physiology and Obstetrics and \\ Gynaecology, University of Western Ontario, London, Ontario, Canada N6A 5 A5
}

\begin{abstract}
Summary. Immature female rats (60-65 g) were injected with 4 i.u. PMSG on Day -2 , and allocated to 3 groups. For Groups I and II, unmated donors were killed $67-69 \mathrm{~h}$ after PMSG injection, shortly after the expected time of ovulation. Oocytes were recovered from the oviducts and transferred immediately into the oviduct of mated recipients (Group I) whose ipsilateral ovary had been exposed by peeling back the bursa, preventing endogenous oocytes from entering the oviduct, or were fertilized in vitro (Group II) and were transferred 16-18 h later. Rats in Group III were allowed to mate and half were killed $6 \mathrm{~h}$ after mating. The fertilized oocytes were then incubated for $10-12 \mathrm{~h}$ until transfer. The remaining rats in Group III were killed $16-18 \mathrm{~h}$ after mating and fertilized oocytes were collected and transferred immediately. Recipient rats were killed on Days 2, 5, 8 and 20.

Zygotes resulting from in-vitro fertilization (Group II) were as able as those fertilized in donors (Group III) or recipients (Group I) to develop to the 2-cell stage, but underwent significantly greater embryonic loss beyond this stage of development. There was a slower rate of development of such oocytes to the blastocyst stage (Day 5) and a lower mean weight of implantation sites (Day 8). Transfer of zygotes after in-vitro fertilization resulted in a loss of $35 \%$ of the embryos at the time of implantation.

These results suggest that in-vitro fertilization of rat oocytes leads to defects in the embryos causing a delay in early embryo development and a large number of implantation losses.
\end{abstract}

\section{Introduction}

Treatment of human infertility by in-vitro fertilization and embryo replacement results in low rates of embryo survival, with over $80 \%$ of transfers failing to establish full-term pregnancies (Grobstein, Flower \& Mendeloff, 1983). Techniques for in-vitro fertilization of rat oocytes have been established (Toyoda \& Chang, 1974; Kaplan \& Kraicer, 1978; Evans \& Armstrong, 1984) and it has been shown that rat oocytes fertilized in vitro are able to develop to fetuses (Toyoda \& Chang, 1974; Shalgi, 1983; Fleming, Evans, Walton \& Armstrong, 1986), although there was a trend for the proportion of oocytes or embryos that developed to Day 20 fetuses to decrease with increasing divergence from the natural situation. Oocytes fertilized in the donor and transferred to a recipient within the next $24 \mathrm{~h}$ exhibited the highest rates of development, while oocytes fertilized in vitro exhibited the lowest rate. Oocytes transferred to a mated recipient immediately after ovulation, for fertilization and development in the recipient, showed an intermediate rate of development (Fleming et al., 1986). It was therefore suggested that in-vitro fertilization or fertilization in the recipient resulted in lower rates of normal embryo development, because of greater susceptibility of unfertilized oocytes to damage incurred during handling or to other environmental stresses.

There are several differences between conditions of fertilization in vivo and in vitro. Although it has been established that greater embryonic loss occurs after in-vitro than after in-vivo fertilization, 
the factor or combination of factors that is responsible for the embryonic loss has yet to be determined. In addition, little is known as to when during development these losses are occurring. Shalgi (1984) has demonstrated that the proportion of in-vivo or in-vitro fertilized oocytes that develop to Day 20 fetuses is only 10\% less than the proportion of viable Day 13 fetuses, suggesting that the embryonic losses due to in-vitro fertilization are occurring before Day 13.

The present set of experiments was designed to compare the development of fetuses to Day 20 after fertilization at different sites, and to determine the time of embryonic loss during the development of oocytes fertilized in vitro.

\section{Materials and Methods}

\section{Animals}

Immature female Sprague-Dawley rats, mature male Sprague-Dawley rats and mature female Long-Evans rats were obtained from Charles River Canada Inc., St Constant, Quebec. They were housed in air-conditioned quarters and allowed free access to food and water. Lighting was provided for $14 \mathrm{~h}$ daily, but the timing of the period of illumination was altered so that ovulation occurred in the early afternoon. All times cited in the text refer to the diurnal cycle of the animals, i.e. $00: 00 \mathrm{~h}$ refers to the midpoint of the dark period and 12:00 $\mathrm{h}$ is the midpoint of the light period.

To clarify the sequence of events of the experimental protocol, the timing of the events is summarized in Fig. 1.

\section{Donors}

At body weight $60-65 \mathrm{~g}$, the immature rats were treated with 4 i.u. PMSG (Equinex: Ayerst, Montreal) at $08: 00 \mathrm{~h}$ on Day -2 (Day $0=$ day of mating). On Day 0 the rats were randomly allocated to 3 groups. Rats in the groups for transfer of unfertilized oocytes (Group I) and of oocytes

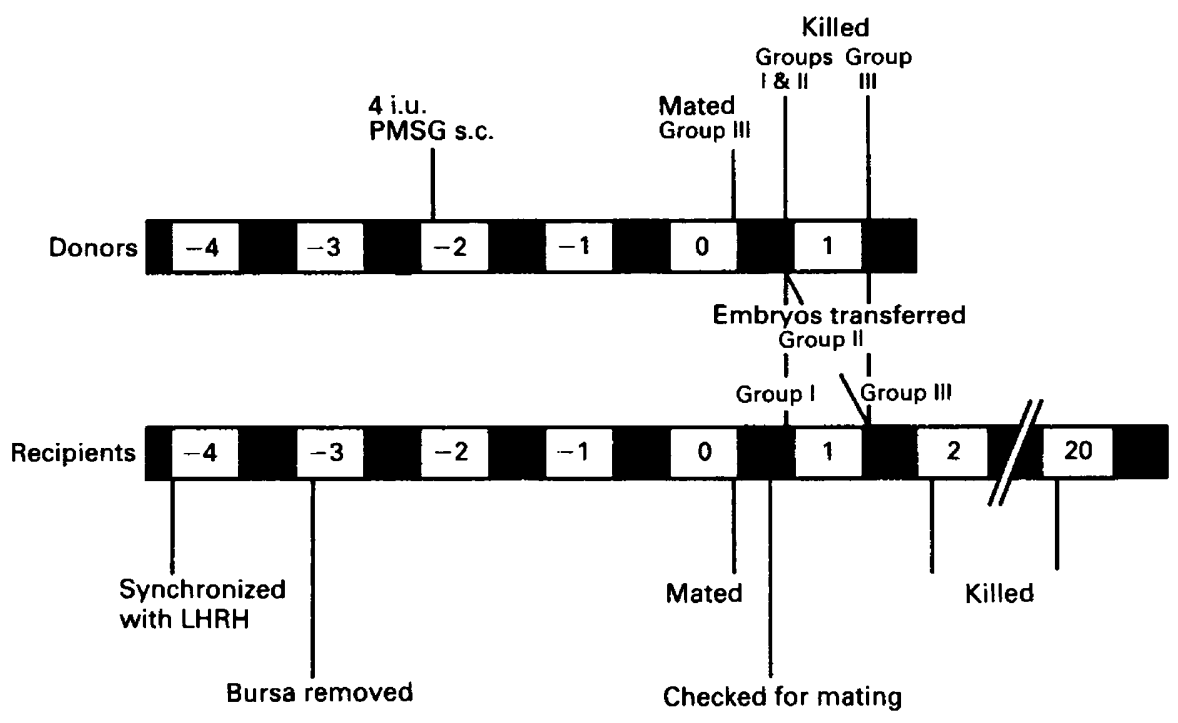

Fig. 1. Diagram of the procedure used for oviducal transfers. Light and dark areas indicate periods of light and dark respectively. The numbers refer to the day of the experiment (Day 1 = first day of pregnancy). 
fertilized in vitro (Group II) were killed on Day 1 between 02:30 and 05:00 h. The oviducts were dissected out in Dulbecco's phosphate-buffered saline (Gibco Laboratories, Grand Island, NY, U.S.A.) with $5 \%$ heat-inactivated, charcoal-treated rat serum (DPBS-S). The swollen ampullae were torn open with fine forceps to allow the oocytes to escape. The oocytes were either fertilized in vitro or transferred to mated recipients (see below). Rats in Group III were caged with males of proven fertility from $20: 00 \mathrm{~h}$ on Day 0 to $02: 00 \mathrm{~h}$ on Day 1, when they were assessed for mating. The presence of a copulatory plug and/or spermatazoa in the vaginal smear was taken as evidence of insemination. Mated donors were killed at 19:00 to 22:00 h on Day I and the ova were flushed from the oviducts with DPBS-S and using a 30-gauge needle. Fertilized ova were collected and transferred immediately (see below).

\section{In-vitro fertilization}

In-vitro fertilization was carried out as described previously (Evans \& Armstrong, 1984) with the following modifications. The sperm preparation was incubated at a concentration of $1.25 \times 10^{6}$ spermatozoa/ml for $1-2 \mathrm{~h}$ before insemination. Oocytes were collected in DPBS-S and transferred to $50 \mu \mathrm{l}$ droplets of fertilization medium, incubated under mineral oil (U.S.P. Heavy, Drug Trading Co., Toronto, Canada) in $10 \times 35 \mathrm{~mm}$ plastic tissue culture dishes (Nunc; Roskilde, Denmark). Each droplet contained 1-6 oocytes and was inseminated 30-90 min later with $40 \mu \mathrm{l}$ sperm suspension to give a final concentration of $5 \times 10^{5}$ spermatozoa/ml. Fertilization of all oocytes in Group II was assessed by phase-contrast microscopy $14-16 \mathrm{~h}$ after insemination. Only ova with 2 pronuclei and 1 sperm tail within the vitellus were transferred. In-vitro and in-vivo fertilized oocytes not transferred were cultured for a further $24 \mathrm{~h}$ period and development to the 2-cell stage was assessed.

\section{Recipients}

Naturally cycling female Long-Evans rats were used, but when synchronization was necessary to ensure an adequate number of mated female rats, the rats were treated with $40 \mu \mathrm{g}$ of an $\mathrm{LHRH}$ agonist (des-Gly ${ }^{10}, \mathrm{D}-\mathrm{Ala}^{6}{ }^{6}$,-ProNHEt ${ }^{9}$ LHRH; Sigma, St Louis, MO U.S.A.) as described previously (Walton \& Armstrong, 1983).

At 3-5 days before the day of transfer, the female rats were anaesthetized with tribromoethanol solution $(2 \%, 0.01 \mathrm{ml} / \mathrm{g}$ body weight, i.p.) and, via a lateral incision, the ovarian bursa of each recipient was peeled back from around the left ovary such that the ovary was completely exposed. This procedure essentially eliminates pick-up of ovulated oocytes by the oviduct, without interfering with ovulation. The effectiveness of this technique was verified in a preliminary study in which unilateral pregnancy was produced in $98 \%(\mathrm{~N}=51)$ of successfully-operated rats.

\section{Transfers}

At 20:00 h of Day 0 , the adult rats were caged with males of proven fertility. The rats were assessed for mating at 02:00 h on Day 1 and only mated rats were subsequently used. Transfers of unfertilized oocytes (Group I) were performed at 02:30 to 05:00 h on Day 1. Oocytes fertilized in the donor (Group III) or in vitro (Group II) were transferred at 19:00-22:00 $\mathrm{h}$ on the same day. The recipient rats were anaesthetized with tribromoethanol solution and the bursa-free (transfer) ovary was drawn out through a lateral incision. Using a finely drawn (150-250 $\mu \mathrm{m}$ i.d.) heat-polished glass pipette, 4 or 5 oocytes or zygotes were transferred to the infundibulum of the left oviduct of each recipient. Volume of transfer material varied between 0.5 and $1 \mu \mathrm{l}$ for cumulus-enclosed oocytes (Group I) to $<0.4 \mu$ for zygotes (Groups II \& III). With the bursa peeled from around the left ovary, the ipsilateral oviduct would contain only transferred oocytes or zygotes. The contralateral horn, containing embryos developing from in-vivo fertilization of the recipients' own 
oocytes, served as a control. Animals with evidence of large adhesions, significant blood clots, or whose peeled bursae had slipped back and enclosed the ovary were not used as recipients. Recipients not pregnant in the control horn were not used in the analyses.

\section{Development of transferred ova}

Experiment 1: effect of site of fertilization. One-third of rats in Group I were killed $16 \mathrm{~h}$ after transfer (18:00 h, Day 1) to assess efficiency of fertilization and transfer. Recipients from all three groups were killed $40 \mathrm{~h}$ after insemination to assess efficiency of transfer and development to the 2cell stage, or at $08: 00 \mathrm{~h}$ on Day 20 to permit estimation of embryonic losses after the first cleavage.

Experiment 2: timing of embryonic mortality after in-vitro fertilization. Zygotes derived from fertilization in vitro or in vivo (Groups II \& III) were transferred. Recipients were killed at 18:00 h on Day $2(40 \mathrm{~h}), 08: 00 \mathrm{~h}$ on Day $5,20: 00 \mathrm{~h}$ on Day 8, or 08:00 h on Day 20.

Experiment 3: effect of incubation of in-vivo fertilized oocytes on development of embryos. To determine possible detrimental effects of incubation on the ability of oocytes fertilized in vivo to develop to Day 20 fetuses, donors (Groups III) were mated as described previously. At $6 \mathrm{~h}$ after mating, one-half of the mated rats were killed and the oocytes were collected and incubated for 10-12 $\mathrm{h}$ before transfer, under conditions similar to those used for in-vitro fertilization, but without the addition of spermatozoa. The remaining mated rats were killed 16-18 h after mating and the ova were collected, assessed for fertilization and the zygotes were transferred immediately. All recipients were killed at $08: 00 \mathrm{~h}$ on Day 20.

\section{Collection of embryos or fetuses}

In all experiments, for animals that were killed on Days 1,2 or 5, both oviducts were removed and flushed with $0.2 \mathrm{ml}$ DPBS-S. On Day 5 , the uterine horns were flushed as well. The recovered oocytes, zygotes and embryos were counted and examined under a phase-contrast microscope to assess the stage of development and the occurrence of fragmentation or other degenerative changes. On Day 8, implantation sites were dissected out, counted and weighed. They were fixed in Bouin's solution and embedded in paraffin wax. Serial sections of $10 \mu \mathrm{m}$ were stained with haematoxylin and eosin and examined for evidence of normal implantation. For animals killed on Day 20, the number of fetuses and the total fetal and placental weights were recorded for the control and transfer horns. The number and size of resorption sites in each horn were also noted.

\section{Statistical analyses}

The proportions of oocytes recovered as 2-cell embryos, blastocysts, implantation sites or fetuses were compared using $\chi^{2}$ analyses with Yates' correction. Comparisons of mean fetal and placental weights in each group were made using one-way analysis of variance with Bartlett's test for homogeneity of variance and Duncan's new multiple range test. In Exp. 3, mean fetal and placental weights in the two groups were compared using a $t$ test (Steel \& Torrie, 1980).

\section{Results}

\section{Experiment 1}

At $16 \mathrm{~h}$ after the transfer of unfertilized oocytes into mated recipients (Group I), $84 \cdot 2 \%(n=38)$ of the oocytes recovered were fertilized. A similar proportion $(85.7 \%, n=84)$ of endogenous oocytes in the control horn were fertilized. At $40 \mathrm{~h}, 77 \cdot 1 \%$ of recovered oocytes were 2-cell embryos, which 
Table 1. Recovery of 2-cell zygotes and Day 20 fetuses after transfer of oocytes or zygotes

\begin{tabular}{|c|c|c|c|c|}
\hline \multirow{2}{*}{$\begin{array}{l}\text { Time } \\
\text { killed }\end{array}$} & \multirow{2}{*}{$\begin{array}{c}\text { Stage of } \\
\text { development }\end{array}$} & \multicolumn{3}{|c|}{$\begin{array}{l}\% \text { (of total no.) of transferred oocytes } \\
\text { or zygotes recovered }\end{array}$} \\
\hline & & Group I & Group II & Group III \\
\hline $16 \mathrm{~h}$ & Zygote & $54 \cdot 3(70)$ & & \\
\hline $40 \mathrm{~h}$ & 2-cell zygote & $46 \cdot 7(75)^{8}$ & $93.9(65)^{b}$ & $73 \cdot 3(45)^{\mathrm{c}}$ \\
\hline Day 20 & Viable fetus & $32 \cdot 9(70)$ & $18 \cdot 2(55)$ & $60 \cdot 0(85)$ \\
\hline Day $20^{*}$ & Viable fetus & $70 \cdot 3(70)^{2}$ & $19 \cdot 3(55)^{\mathrm{b}}$ & $81.9(85)^{\mathrm{a}}$ \\
\hline Day 20 & Resorption site & $10 \cdot 0(70)^{\mathrm{a}}$ & $36 \cdot 4(55)^{b}$ & $14 \cdot 1(85)^{a}$ \\
\hline
\end{tabular}

* Corrected for losses before $40 \mathrm{~h}$ after transfer.

Within each time, values with different superscripts are significantly different $(P<0 \cdot 01)$.

was significantly lower $(P<0.05)$ than the percentage of 2 -cell embryos recovered after transfer of zygotes following fertilization in vivo $(93.9 \%, n=49)$ or in vitro $(98.4 \%, n=61)$ (Groups III \& II). However, when adjusted for the $84 \%$ fertilization rate, the percentage of 2-cell embryos recovered after transfer of unfertilized oocytes increased to $91.5 \%(n=35)$, which was not significantly different from the values for the other two groups.

Pronuclear ova resulting from in-vitro and in-vivo fertilization and not transferred, but cultured for a further $24 \mathrm{~h}$ period, showed statistically similar in-vitro cleavage rates of $89.9 \%$ $(n=109)$ and $98.2 \%(n=56)$, respectively.

The proportion of transferred oocytes or zygotes recovered $40 \mathrm{~h}$ after insemination was significantly different between all three groups $(P<0.01)$ (Table 1$)$. The percentage of transferred oocytes or zygotes recovered on Day 20 reflected, in part, losses that occurred due to the transfer technique or to failure of fertilization. To indicate more accurately the developmental abilities of the oocytes, these numbers were corrected for the losses occurring before Day 2. After this correction, less than $20 \%$ of zygotes produced by in-vitro fertilization were recovered as live fetuses. This low percentage of recovery on Day 20 was associated with a significantly higher $(P<0.005)$ percentage of resorption sites, compared to Groups I and III. About $90 \%$ of all resorption sites were $<6 \mathrm{~mm}$ in diameter.

\section{Experiment 2}

There were notable differences between Groups II and III with respect to the stage of development at which the transferred zygotes were lost (Table 2). Zygotes transferred after in-vivo fertilization were lost primarily during the $24 \mathrm{~h}$ after transfer $(26 \%)$ with no significant loss between Days 2 and 20 . In contrast, $24 \%$ of the in-vitro fertilized oocytes were lost during the development from 2-cell zygotes to morulae or blastocysts, with further losses occurring after the blastocyst stage $(40 \%)$.

In each group the percentage of recovered zygotes that had cleaved to 2 cells was comparable to that in Exp. 1, with $89.7 \%$ of in-vitro fertilized oocytes and $100.0 \%$ of in-vivo fertilized oocytes developing to the 2-cell stage $(P>0.05)$.

Embryos produced by in-vivo fertilization and recovered on Day 5 were primarily at the blastocyst stage of development, although a small percentage were still at the morula stage (Table 3). These results were not statistically different from the development of embryos in the control horns of both groups. In contrast, more than half of the embryos produced in Group II were developing at a slower rate than those in the control horn. In both groups, $<3 \%$ of the recovered embryos were degenerate. 
Table 2. Recovery of embryos at 2, 5, 8 and 20 days after the transfer of in-vivo or in-vitro fertilized oocytes

\begin{tabular}{llll}
\hline & \multicolumn{1}{c}{$\begin{array}{c}\text { Stage of } \\
\text { Time } \\
\text { killed }\end{array}$} & \multicolumn{1}{c}{$\begin{array}{c}\text { (otal no.) of zygotes } \\
\text { recovered }\end{array}$} \\
\hline Day 2 & 2-cell zygotes & Group II & Group III \\
Day 5 & Blastocysts/morulae & $86 \cdot 7(45)^{\mathbf{a}}$ & $74 \cdot 0(50)^{\mathrm{a}}$ \\
Day 8 & $\begin{array}{l}\text { Implantation/ } \\
\text { decidualization sites }\end{array}$ & $62 \cdot 4(85)^{\mathrm{a}}$ & $67 \cdot 7(65)^{\mathrm{a}}$ \\
Day 20 & Fetuses & $57.5(80)^{\mathrm{a}}$ & $62.9(70)^{\mathrm{a}}$ \\
\hline
\end{tabular}

a,b Within each time, values with different superscripts are significantly different $(P<0.001)$.

Table 3. Development of transferred zygotes to Day 5 embryos

\begin{tabular}{llll}
\hline & & \multicolumn{2}{c}{$\begin{array}{c}\% \text { (of total no.) of zygotes } \\
\text { recovered }\end{array}$} \\
\cline { 3 - 4 } & $\begin{array}{c}\text { Stage of } \\
\text { development }\end{array}$ & Group II & Group III \\
\hline Control horn & Blastocyst & $89 \cdot 2(93)^{\mathrm{a}}$ & $86 \cdot 3(73)^{\mathrm{a}}$ \\
& Morula & $-8 \cdot 6(93)^{\mathrm{c}}$ & $13 \cdot 7(73)^{\mathrm{c}}$ \\
Transfer horn & Blastocyst & $45 \cdot 3(53)^{\mathrm{b}}$ & $88 \cdot 6(44)^{\mathrm{a}}$ \\
& Morula & $52 \cdot 8(53)^{\mathrm{d}}$ & $-9 \cdot 1(44)^{\mathrm{c}}$ \\
\hline
\end{tabular}

Values with different superscripts for each developmental stage are significantly different $(P<0.001)$.

On Day 8 , there was a significant difference $(P<0.05)$ between the mean weights $(\mathrm{g})$ of the implantation sites in rats in Groups II and III (Group II: control horn, $36 \cdot 0 \pm 1 \cdot 1, n=16$; transfer horn, 29.4 $\pm 1 \cdot 8, n=15$; Group III: control horn, $33 \cdot 9 \pm 2 \cdot 2, n=12$; transfer horn, $35 \cdot 2 \pm 1 \cdot 4$, $n=13$ ). Histological examination of the implantation sites from the transfer horn in Group II rats revealed that $60 \%$ of the 20 sites examined showed no evidence of an embryo or trophoblastic invasion, suggesting that the embryos elicited a decidual response but developed no further. The remaining $40 \%$ of sites and those from the transfer horn in Group III $(n=16)$ and the control horns in both groups $(n=27)$ all contained embryos with evidence of implantation.

The percentage of transferred zygotes that developed to Day 20 fetuses in this experiment (Table 2) was comparable to the recoveries in Exp. 1. Associated with the great embryonic losses in Group II was a significantly higher $(P<0.001)$ percentage of resorption sites $(32.7 \%)$; only $10 \%$ of Group III zygotes were recovered as resorption sites. All resorption sites were $6 \mathrm{~mm}$ or less in diameter.

\section{Experiment 3}

The recovery of viable fetuses after transfer of in-vivo fertilized oocytes with or without in-vitro incubation was not significantly different $(68.5 \%, n=92$, and $62.8 \%, n=43$, respectively). There was also a statistically similar proportion of resorbing fetuses in both groups $(18.5 \%, n=92$, and $11 \cdot 6 \% n=43$, respectively).

The mean fetal and placental weights combined for all experiments are summarized in Table 4. There was no significant difference between experiments in any of the values measured. There were 
Table 4. Mean fetal and placental weights 20 days after oocyte/zygote transfer

\begin{tabular}{lccccc}
\hline & \multicolumn{2}{c}{ Fetal weight $(\mathrm{g})$} & & \multicolumn{2}{c}{ Placental weight (mg) } \\
\cline { 2 - 3 } \cline { 5 - 6 } Group & Control side & Transfer side & & Control side & Transfer side \\
\hline I & $2.22 \pm 0.05(13)^{\mathrm{a}}$ & $2.00 \pm 0.07(10)^{\mathrm{a}}$ & & $489 \pm 12(12)^{\mathrm{a}}$ & $592 \pm 50(9)^{\mathrm{b}}$ \\
II & $2.07 \pm 0.05(20)^{\mathrm{a}}$ & $1.79 \pm 0.11(11)^{\mathrm{a}}$ & & $485 \pm 16(20)^{\mathrm{a}}$ & $510 \pm 44(11)^{\mathrm{a}}$ \\
III & & & & \\
$\quad$ No incubation & $2.02 \pm 0.05(31)^{\mathrm{a}}$ & $1.90 \pm 0.05(31)^{\mathrm{a}}$ & & $465 \pm 12(31)^{\mathrm{a}}$ & $456 \pm 13(31)^{\mathrm{a}}$ \\
Incubation $^{*}$ & $2.05 \pm 0.03(19)^{\mathrm{a}}$ & $1.98 \pm 0.04(19)^{\mathrm{a}}$ & & $454 \pm 11(19)^{\mathrm{a}}$ & $451 \pm 15(19)^{\mathrm{a}}$ \\
\hline
\end{tabular}

* For $10-12 \mathrm{~h}$.

Values are mean \pm s.e.m. for the number of rats in parentheses.

Values with different superscripts are significantly different $(P<0.005)$, within columns and between relevant columns.

no significant differences in the mean fetal and placental weights in the control horn, although Bartlett's test indicated heterogeneity of variance amongst the fetal weights. In the transfer horn there was no significant difference between the mean fetal weights of the four groups. However, mean placental weight of the fetuses in Group I was significantly greater than those in Group II and Group III $(P<0.005)$.

\section{Discussion}

The experiments reported here have examined a number of the potential factors that may be responsible for the reduced developmental abilities of embryos produced by in-vitro fertilization. It is clear that handling of the unfertilized oocyte with subsequent fertilization in vivo had no detrimental effect on the development of the oocyte. Once fertilized, the oocytes in Group I were able to develop into 2-cell embryos or Day 20 fetuses in proportions comparable to oocytes that were fertilized in the donor and then transferred at the pronuclear stage (Group III).

Transfer of unfertilized oocytes to mated recipients for in-vivo fertilization gave a significantly lower rate of recovery of fetuses than did transfer of oocytes fertilized in the donor. When corrected for losses within $40 \mathrm{~h}$ of transfer, the rate of recovery indicated that only a small portion of the loss of embryos occurred after the 2-cell stage of development, i.e. the proportion of 2-cell embryos that developed to Day 20 fetuses was similar in both groups after in-vivo fertilization. The low recovery of 2-cell embryos at $40 \mathrm{~h}$ was not significantly different from the percentage of ova recovered $16 \mathrm{~h}$ after transfer, suggesting that embryo loss following transfer of unfertilized oocytes may be at least partly due to losses of the oocytes shortly after transfer. When oocytes are collected immediately after ovulation and transferred, the presence of cumulus cells necessitates the use of a larger bore pipette and a larger volume of medium, which has been suggested to increase losses due to regurgitation (Shalgi, Dekel \& Kraicer, 1979).

The results of the experiments presented here show that, compared to oocytes fertilized in vivo, a lower proportion of oocytes fertilized in vitro develop to viable fetuses. A comparable decline in development was observed when in-vitro fertilized oocytes were transferred at the 2-cell stage of development (Shalgi, 1983). By recovering embryos on Day 2, the present study determined that there was no deficiency in the ability of in-vitro fertilized oocytes to undergo the first cleavage division. With correction for losses before Day 2, it is evident that the majority of the embryonic losses occurred after the 2-cell stage of development, which is in contrast to the time of loss of oocytes transferred before fertilization. This finding is supported by the number of resorption sites that are seen on Day 20. The increased number of resorption sites found in Group II suggests that some of the losses occurred at or after implantation. 
Shalgi (1984) indicated that $34 \%$ of in-vitro fertilized rat zygotes developed to Day 13 fetuses, when examined by laparotomy. The present study demonstrates that a fairly high percentage $(58 \%)$ of zygotes derived from in-vitro fertilization will develop to embryos capable of eliciting decidualization, but a considerable proportion of these undergo no further development. The slower rate of development of such zygotes to the blastocyst stage suggests a possible reason for the high rate of unsuccessful implantation in Group II. When the embryo is delayed in development, as it was in $53 \%$ of the embryos from in-vitro fertilization, implantation may or may not occur, depending on the degree of asynchrony in development of the embryo and the uterine environment (Dickmann \& Noyes, 1960; Mantalenakis \& Danezis, 1968). Since a variety of artificial physical stimuli can increase endometrial vascular permeability and bring about decidualization (Psychoyos, 1973), it is possible that, during the period of optimal endometrial sensitivity, the physical presence of the embryo, even at an earlier stage of development, may be sufficient to initiate the decidual cell reaction, without subsequent implantation. This postulate is supported by the results of the histological examination of the uterine swellings. For example, some of what were recorded in this study as implantation sites on Day 8 were simply sites of decidualization in the absence of implantation. The lower mean weight of the implantation sites in Group II, as compared to that in Group III, is indicative of the higher proportion of decidualization sites as opposed to implantation sites. The majority of the resorption sites seen on Day 20 (i.e. those $<6 \mathrm{~mm}$ ) were probably necrotic masses that resulted from the regression of these deciduomata (Lobel, Tic \& Shelesnyak, 1965).

In the present study the majority of the embryonic losses after in-vitro fertilization occurred after the 2-cell stage of development. As there was no evidence for developmental inability after in-vivo fertilization, it may be concluded that embryo losses beyond the 2-cell stage of development are due to defects in the embryos produced by in-vitro fertilization. Losses might be due to the absence, in culture, of some factor provided in vivo during the $16 \mathrm{~h}$ after fertilization, or culture conditions may have had a detrimental effect which was not manifested in the zygote until later stages of development. The results of Exp. 3 indicate that, after fertilization in vivo, handling of zygotes, incubation or exposure to environmental stresses such as light or changes in temperature do not have any detrimental effects on the development to Day 20 fetuses.

During in-vitro fertilization, the conditions for gamete interaction vary considerably from conditions in vivo. Exposure of the oocytes to a large number of spermatozoa leads to relatively high rates of polyspermic fertilization (Niwa \& Chang, 1975). In this experiment oocytes fertilized in vitro and intended for transfer were examined and only those with two pronuclei and one sperm tail within the vitellus were transferred. These criteria were insufficient, however, to assess the developmental abilities of the zygotes. Shalgi \& Phillips (1982) have demonstrated a different morphological pattern of sperm attachment and penetration into oocytes in vitro compared to in vivo. It is clear that further investigation is needed into the relevance of this difference, and others resulting from in-vitro conditions, in the development of the zygotes.

Development of fetuses after the transfer of unfertilized oocytes into mated recipients was associated with placental weights that were greater than those of the other groups. Although it was not significant, placentas associated with fetuses developing from oocytes fertlized in vitro were also heavier. The reason for these elevated weights is not known, but it may be associated with the fact that generally there are fewer fetuses developing in the uterine horn of rats in these two groups.

The similarity in the percentage survival of fetuses developing from rat oocytes fertilized in vitro in our studies and the overall pregnancy rates generally reported in the most successful human in-vitro fertilization programmes (Grobstein et al., 1983) suggests that similar mechanisms may be operating to cause the high rate of post-fertilization embryonic losses in both species.

This work was supported by the M.R.C. of Canada; D.T.A. is a Career Investigator of the M.R.C. We thank Ms J. Rutledge for excellent technical assistance. 


\section{References}

Dickmann, Z. \& Noyes, R.W. (1960) The fate of ova transferred into the uterus of the rat. J. Reprod. Fert. 1, 197-212.

Evans, G. \& Armstrong, D.T. (1984) Reduction in fertilization rate in vitro of oocytes from immature rats induced to superovulate. J. Reprod. Fert. 70, 131-135.

Fleming, A.D., Evans, G., Walton, E.A. \& Armstrong, D.T. (1986) Developmental capability of rat oocytes matured in vitro in defined medium. Gamete Res. (in press).

Grobstein, C., Flower, M. \& Mendeloff, J. (1983) External human fertilization: an evaluation of policy. Science, N.Y. 222, 127-133.

Kaplan, R. \& Kraicer, P.F. (1978) Effect of elevated calcium concentration on fertilization of rat oocytes in vitro. Gamete Res. 1, 281-285.

Lobel, B.L., Tic, L. \& Shelesnyak, M.C. (1965) Studies on the mechanism of nidation. XVII. Histochemical analysis of decidualization in the rat. Part 4: Regression of deciduomata. Acta endocr., Copenh. 50, 537-559.

Mantalenakis, S.J. \& Danezis, J.M. (1968) Effect of blastocyst transfer on gestation period in rats. Fert. Steril. 19, 910-913.

Niwa, K. \& Chang, M.C. (1975) Fertilization of rat eggs in vitro at various times before and after ovulation with special reference to fertilization of ovarian oocytes matured in culture. J. Reprod. Fert. 43, $435-451$.
Psychoyos, A. (1973) Endocrine control of egg implantation. In Handbook of Physiology; Section 7, Vol. 2, Part 2, pp. 187-215. Eds R. O. Greep, E. B. Astwood \& S. R. Geiger. Physiological Society, Washington, D.C.

Shalgi, R. (1983) Success of rat embryos derived from in vitro as compared to in vivo fertilization. Biol. Reprod. 28, Suppl. 1, 40, Abstr.

Shalgi, R. (1984) Developmental capacity of rat embryos produced by in vivo or in vitro fertilization. Gamete Res. 10, 77-82.

Shalgi, R. \& Phillips, D.M. (1982) Sperm penetration into rat ova fertilized in vitro. J. Androl. 3, 382-387.

Shalgi, R., Dekel, N. \& Kraicer, P.F. (1979) The effect of LH on the fertilizability and development capacity of rat oocytes matured in vitro. J. Reprod. Fert. 55, 429-435.

Steel, R.G.D. \& Torrie, J.H. (1980) Principles and Procedures of Statistics. McGraw-Hill, Inc., New York.

Toyoda, Y. \& Chang, M.C. (1974) Fertilization of rat eggs in vitro by epididymal spermatozoa and the development of eggs following transfer. J. Reprod. Fert. 36, 9-22.

Walton, E.A. \& Armstrong, D.T. (1983) Oocyte normality after superovulation in immature rats. J. Reprod. Fert. 67, 309-314. 